werden. Sachtleben ridmet der Bestäubungsmethode nur wenig Raum. Ei verweist auf die während der Drucklegung erschienene Schrift von K. Escherich, Die Flugzeugbekïmpfung gegen Forstschädlinge", (Flngschriften der Dt. Ges. f. angew. Entomologie, Nr. 12, Berlin 1929), in der alle theoretischen und praktischen Fragen der Flugzeugbekämpfung eingehend behandelf sind. Es folgen noch einige Angaben über biologische Bekämpfung und waldbauliche Maßnahmen, utnd die Monographie sehließt mit einem Verzeichnis der gesamten zitierten Jiteratur. H. Eidmann, Hann. Münden.

Untersuchungen über Blausäurevergasung. Von Dr. Rasch.

Wagoner, C. C., Calciam Cyanide Dust for Rosy Apple Aphis. Journ. econ, entom. 1924.

Zehn etwa 25 Jahre alte von Blattläusen befallene Apfelläume wurden im Jahre 1923, als die jungen Früchte etwa $3 \mathrm{~cm}$ groß und die Blätter bereits gekräuselt waren, zwischen 14 und $16 \mathrm{Uhr}$ mit je etwa 3 liss. Zyanogas, das mit $75 \%$ Talkum gemischt war, mittels eines Handzerstäubers bestäubt. Das Wetter war seit mehreren Tagen trocken, schwacher Wind, $34^{\circ}$, $85^{\circ}$ Feuchtigkeit. Fünf Hinuten nach Zerstäubung der: Menge wurden die Bäume geschüttelt und etwa 3 cbzoll tote Insekten aufgesammelt. Zehn Stunden später fand eine zweite Priifung statt, die ergab, daß alle Insekten ahgetötet waren. Die laufende Beobachtung während der Vegetationsperiode zeigte, daß weder an Blättern noch an Frichten irgend ein Schaden eingetreten war.

\section{*}

Violle, H., Deuxième rapport sur la dératisation des navires par l'acide cyanhydrique. Revne dhyg. 1926 , (48), S. $800-803$.

Es wird über die Vorführung eines von $L(a$ ainé) in Marseille konstruierten Gasgenerators (Sanosverfahren) berichtet, mit dessen Hilfe die Durchgasung eines Schiffsladeraumes mittlerer Größe in drei Stunden geschehen kann: 10-15 Minuten für die Beschickung, eine Stunde Einwirkungszeit, zwei Stunder Lüftung. Dabei sind zufriedenstellende Eifolge in der Abtötung von Ratten und Insekten bei einer Konzentration von $2 \mathrm{~g} / \mathrm{m}^{3}$ Blausäurt zu erzielen. Nach der Torführung sollte der Apparat durch den Verf. im Auftrage des Gesundheitsrates geprüft werden. Terf. ist der Ansicht, daß der Apparat sich ausgezeichnet bewihrt habe und daß er sehr sicher und zuverlässig sei. Bei Anwendung eines Tentilator's von $4000 \mathrm{~m}^{3}$ Stundenleistung kann die Lüftungszeit sogar auf eine Stunde herabgesetzt werden. In dieser Hinsicht schlägt das Blausäureverfahren alle anleren Verfahren, die infolge der längeren zu ihrer Ausführung gebrauchten Zeit kostspieliger sind. Auf Grund der Tersuche und des erstatteten Berichtes wird für die .lıwendung des Aplarates bei Schiffsdurchgasungen in Frankreich die nachgesuchte Genelımigung erteilt.

\title{
Mitteilungen der Deutschen Gesellschaft für angew. Entomologie E.V. MITGLIEDERVERSAMMLUNG 1930.
}

In diesem Jahre findet eine MITGLIEDERVERSAMMLUNG der Deutschen Gesellschaft für angewandte Entomologie und zwar in der Zeit vom 29. August bis 2. September in Rostock statt. Der Zeitpunkt ist so gewählt, daß die Teilnehmer bequem zu der einige Tage später in Königsberg stattfindenden Versammlung deutscher Naturforscher und Ärzte reisen können.

Es sollen vor allem epidemiologische Fragen und sodann Fortschritte der technischen Bekämpfung behandelt werden.

Anmeldungen zu Vorträgen nimmt der Schriftführer entgegen.

Nähere Angaben werden in einer der nächsten Nummern des Anzeigers erfolgen; außerdem wird den Mitgliedern zur rechten Zeit ein ausführ. liches Programm zugehen.

\section{I T G LIEDSBE I T R Ä GE.}

Die Mitglieder werden ersucht, die Jahresbeiträge einsenden zu wollen. ZAHLSTELLE: Commerz- und Privatbank, Filiale GieBen (Hessen).

Der Beitrag für persönliche Mitglieder ist auf RM 10,-, für korporative z. B. Firmen auf RM 30,- festgesetzt. Der Schriftführer: F. Stellwaag. 